\title{
Article
}

\section{Carbon-Dot-Sensitized, Nitrogen-Doped TiO2in Mesoporous Silica for Water Decontamination through Nonhydrophobic Enrichment-Degradation Mode}

Cheng, Chen, Tan, Xianjun, Lu, Deli, Wang, Lingzhi, Sen, Tapas, Lei, Juying, El-Toni, Ahmed Mohamed, Zhang, Jinlong, Zhang, Fan and Zhao, Dongyuan

Available at http://clok.uclan.ac.uk/13667/

Cheng, Chen, Tan, Xianjun, Lu, Deli, Wang, Lingzhi, Sen, Tapas ORCID: 00000002-0463-7485, Lei, Juying, El-Toni, Ahmed Mohamed, Zhang, Jinlong, Zhang, Fan et al (2015) Carbon-Dot-Sensitized, Nitrogen-Doped TiO2in Mesoporous Silica for Water Decontamination through Nonhydrophobic Enrichment-

Degradation Mode. Chemistry - A European Journal, 21 (49). pp. 17944-17950. ISSN 1521-3765

It is advisable to refer to the publisher's version if you intend to cite from the work. http://dx.doi.org/10.1002/chem.201502301

For more information about UCLan's research in this area go to http://www.uclan.ac.uk/researchgroups/ and search for <name of research Group>.

For information about Research generally at UCLan please go to http://www.uclan.ac.uk/research/

All outputs in CLoK are protected by Intellectual Property Rights law, including Copyright law. Copyright, IPR and Moral Rights for the works on this site are retained by the individual authors and/or other copyright owners. Terms and conditions for use of this material are defined in the policies page. 


\title{
Carbon-dot Sensitized and $\mathrm{N}$-doped $\mathrm{TiO}_{2}$ in Mesoporous Silica for Water Decontamination through Non-hydrophobic Enrichment- Degradation Mode
}

\author{
Chen Cheng ${ }^{1}$, Lingzhi Wang ${ }^{1,2 *}$,Tapas Sen, ${ }^{3}$ Juying Lei, ${ }^{1}$ Ahmed Mohamed El-Toni ${ }^{4,5}$, Jinlong \\ Zhang, ${ }^{1 *}$ Fan Zhang ${ }^{2 *}$ and Dongyuan Zhao ${ }^{2}$
}

\begin{abstract}
Mesoporous silica synthesized from the co-condensation of tetraethoxysilane and silylated carbon dot containing amide group has been adopted as the carrier for the in-situ growth of $\mathrm{TiO}_{2}$ through an impregnation-hydrothermal crystallization process. Benefitted from the initial complexing between the titania precursor and carbon dot, highly dispersed anatase $\mathrm{TiO}_{2}$ nanoparticles can be formed inside the mesoporous channel. The hybrid material possesses ordered hexagonal mesostructure with a $p 6 \mathrm{~mm}$ symmetry, high specific surface area $\left(446.27 \mathrm{~m}^{2} \mathrm{~g}^{-1}\right)$, large pore volume $\left(0.57 \mathrm{~cm}^{3} \mathrm{~g}^{-1}\right)$, uniform pore size $(5.11 \mathrm{~nm})$ and a wide absorption band between $300-550 \mathrm{~nm}$. $\mathrm{TiO}_{2}$ nanocrystals are anchored to carbon dot through bonds of $\mathrm{Ti}-\mathrm{O}-\mathrm{N}$ and $\mathrm{Ti}-\mathrm{O}-\mathrm{C}$ as revealed by $\mathrm{X}$-ray photoelectron spectroscopy. Moreover, the nitrogen doping of $\mathrm{TiO}_{2}$ is also verified by the formation of $\mathrm{Ti}-\mathrm{N}$ bond. This composite shows excellent adsorption capability to organic 2, 4dichlorophenol and acid orange 7 with electron-deficient aromatic ring through the electron donor-acceptor interaction between carbon dot and organics instead of hydrophobic effect as analyzed by the contact angle analysis, which can be photocatalytically recycled through visible light irradiation after the adsorption. The narrowed bandgap by nitrogen doping and the photosensitization effect of carbon dot are revealed to be co-responsible for the visible-light activity of $\mathrm{TiO}_{2}$. The adsorption capacity does not suffer obvious loss after being recycled 3 times.
\end{abstract}

\section{Introduction}

$\mathrm{TiO}_{2}$ semiconductor with good chemical stability has drawn most attention as photocatalysts for new energy development and pollutant degradation ${ }^{[1]}$. Nanometer-sized $\mathrm{TiO}_{2}$ has superior photocatalytic activity to bulk counterpart since it has more surface-active sites and higher electron-hole separation rate ${ }^{[2]}$. Many efforts have been devoted to decrease the particle size. However, the side effects of size-reduction lie in that nanometer-

Key Lab for Advanced Materials and Institute of Fine Chemicals, East China University of Science and Technology, Shanghai, 200237, P. R. China

E-mail: wlz@ecust.eud.cn; jlzhang@ecust.edu.cn

2 Department of Chemistry and Laborotary of Advanced Materials, iChEm (Collaborative Innovation Center of Chemistry for Energy Materials), State Key Laboratory of Molecular Engineering of Polymers, Fudan University, Shanghai 200433, P. R. China E-mail: zhang_fan@fudan.edu.cn

3 Center for Materials Science, Institute of Nanotechnology and Bioengineering, School of Forensic and Investigative Sciences, University of Central Lancashire, Preston

$4 \quad$ King Abdullah Institute for Nanotechnology, King Saud University, Riyadh 11451, Saudi Arabia

5 Central Metallurgical Research and Development Institute, CMRDI, Helwan 11421, Cairo, Egypt

Supporting information for this article is given via a link at the end of the document. sized $\mathrm{TiO}_{2}$ have strong agglomeration tendency and narrowed light-absorption range due to the quantization-size effect. ${ }^{[3]}$ To solve the above ubiquitous contradictions encountered during the photocatalysis application, intensive studies about loading photocatalyst on carrier with large specific surface area, and doping or sensitization of $\mathrm{TiO}_{2}$ to extend the light absorption range of $\mathrm{TiO}_{2}{ }^{[4]}$ have been respectively or jointly carried out.

Mesoporous silica with a large specific surface area, modifiable framework surface and tunable pore size from 2-50 $\mathrm{nm}$ has proven to be good carriers for the loading of photocatalysts by versatile methods including co-assembly, insitu growth and post-impregnation ${ }^{[5]}$. Additional treatment has also been utilized to activate loaded $\mathrm{TiO}_{2}$ in visible light range. For examples, $\mathrm{Ti}-\mathrm{O}-\mathrm{Cr}^{[6]}$, $\mathrm{Ti}-\mathrm{O}-\mathrm{Ce}^{[7]}$ have been formed in mesoporous silica to improve the visible light activity. Moreover, in consideration of the abundant porous system, mesoporous silica can simultaneously play the role of pollutant adsorption ${ }^{[8]}$, improving the photocatalytic activity through the pollutantenrichment effect. To achieve the cooperative effect between adsorption and photocatalysis, the reasonable spatial arrangement and uniform dispersion of adsorption and photocatalysis sites are essential, which however has not got efficiently solved due to the great reactivity of most titania precursors with low electronegativity and the high coordination numbers ${ }^{[9]}$. Uncontrollable growth and local aggregation have been commonly observed, which discount the cooperative effect of adsorption and photocatalysis.

Here, we adopt a silylated carbon dot $(C D)^{[10]}$ composed of graphitic core and amide-covered surface for the formation of mesoporous silica through the co-condensation of $\mathrm{CD}$ and tetraethoxysilane (TEOS). Through the complexing of amide groups with $\mathrm{Ti}\left(\mathrm{SO}_{4}\right)_{2}$ precursor and a hydrothermal crystallization process, highly dispersed $\mathrm{TiO}_{2}$ are subsequently in-situ grown in the pore channels without sacrificing the pore accessibility. $\mathrm{TiO}_{2}$ nanoparticles are nitrogen-doped and bridged to $\mathrm{CD}$ through Ti-O-C and Ti-O-N bonds. This composite shows high adsorption efficiency to 2, 4-dichlorophenol (2, 4-DCP) and azo dye acid orange 7 (AO7) through electron donor-acceptor interaction between $C D$ and organics. The enriched organics around $\mathrm{TiO}_{2}$ can be photocatalytically degraded under visible light irradiation. The visible-light activity is attributed to the coeffect of nitrogen-doping and CD sensitization. The adsorptiondegradation process can be recycled 3 times without causing obvious loss of adsorption capacity.

\section{Results and Discussion}

CD is formed from the pyrolysis reaction of citric acid in amine-containing silane ${ }^{[10]}$. The product sealed in hexane is sticky and yellowish brown and the film spin-coated from the hydrolyzed CD shows yellow color (Fig. 1A). The UV-Vis diffuse reflectance spectrum of $C D$ film shows a wide absorption band 
from 300 to $550 \mathrm{~nm}$. (Fig. $1 \mathrm{~B}$ ). The Fourier transform infrared (FTIR) spectrum shows bands at 1520-1650, 960-1140 and 698$860 \mathrm{~cm}^{-1}$ ascribed to the vibrations of $\mathrm{CONH}_{2}$, Si-O and $\mathrm{Si}-\mathrm{CH}_{2}$ groups, respectively (Fig. 1C). The high-resolution transmission electron microscopy (HRTEM) shows that CD is monodispersed and polycrystallized with particle size of 1-2 $\mathrm{nm}$ (Fig. $1 \mathrm{D}$ ).

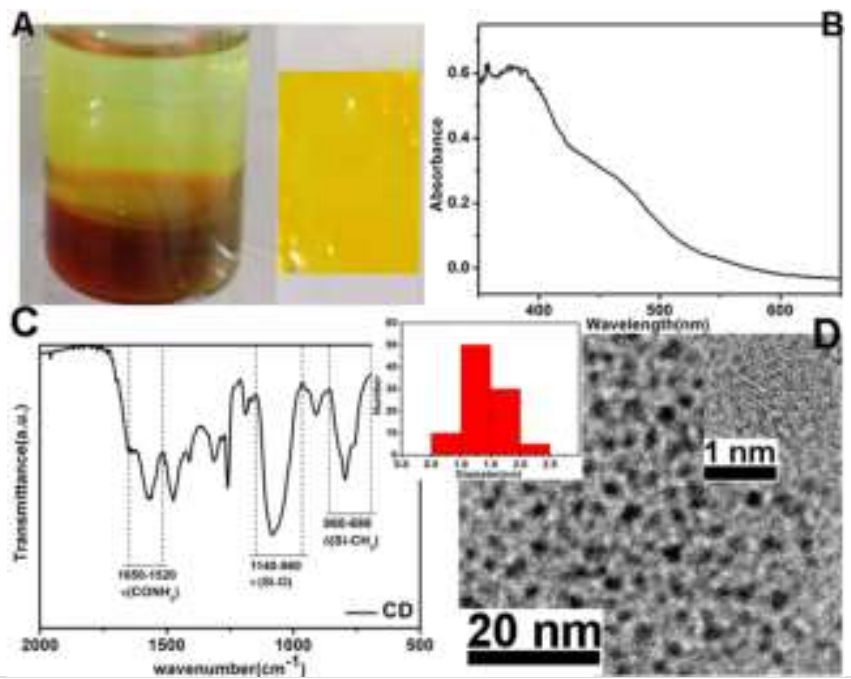

Figure 1. Optical photographs of the hexane solution of CD (left) and the film obtained by spin-coating the hydrolysed CD on glass slide (right); (B)-(D) UVVis diffuse reflectance and FTIR spectra and HRTEM image of CD nanoparticles. Insert: Histogram of the particle diameter distribution (Left) and the enlarged HRTEM image (Right) of CD.

CD-embedded mesoporous silica (CD-MS) was synthesized according to our previous report, which uses CD and TEOS as the co-precursor of silica and $\mathrm{EO}_{20} \mathrm{PO}_{70} \mathrm{EO}_{20}$ (P123) as the template ${ }^{[10 \mathrm{~d}]} . \mathrm{TiO}_{2}$ nanoparticles were subsequently in-situ formed on CD-MS using $\mathrm{Ti}\left(\mathrm{SO}_{4}\right)_{2}$ as the titania precursor through the process of impregnation and hydrothermal crystallization (Ti-CD-MS). TEM images indicate that highly dispersed $\mathrm{TiO}_{2}$ nanocrystals (ca. $4 \mathrm{~nm}$ ) can be formed in the pore channel of CD-MS (Fig. 2 A), while severely local aggregation occurs for $\mathrm{TiO}_{2}$ particles grown on MS (Ti-MS, Fig. $2 \mathrm{~B}$ ), indicating the formation of uniformly distributed $\mathrm{TiO}_{2}$ nanocrystals on CD-MS should be related to the existence of $C D$ in the pore channel. It is known that chelating agent with mercapto, amine or carbonyl group is advantageous to inhibit the growth of metal or metal oxide nanoparticles through forming metal ion complex ${ }^{[11]}$. Therefore, the amide group of CD should be responsible for suppressing the severe hydrolysis of titania precursor and the subsequent agglomeration of $\mathrm{TiO}_{2}$. The wideangle X-ray powder diffraction (XRD) pattern shows that $\mathrm{TiO}_{2}$ nanocrystal has anatase phase and the low intensity is ascribed to the tiny size of anatase $\mathrm{TiO}_{2}$ (Fig. $2 \mathrm{C}$ ). The small-angle XRD pattern shows a peak at $2 \theta=0.834^{\circ}$ ascribed to 10 reflection of a 2D hexagonal mesostructure with a space group of $p 6 \mathrm{~mm}^{[5 \mathrm{~b}]}$, indicating that the mesostructure is still retained after the introduction of $\mathrm{TiO}_{2}$ (Fig. 2 D). The $\mathrm{N}_{2}$ adsorption-desorption isotherms (Fig. $2 \mathrm{E}$ ) indicates this hybrid material has typical type IV curves with sharp capillary condensation steps in the middle $P / P_{0}$ range. The specific surface area is calculated to be
$446.27 \mathrm{~m}^{2} \mathrm{~g}^{-1}$ by Brunauer-Emmett-Teller (BET) method. A narrow pore-size distribution curve with the mean value of $\sim 5.11$ $\mathrm{nm}$ is obtained from the adsorption branch using Barrett-Joyner-Halenda (BJH) model (Fig. 2 F). The pore volume is about $0.57 \mathrm{~cm}^{3} / \mathrm{g}$. These data are comparable to those of CD-MS without embedding of $\mathrm{TiO}_{2}$ (Fig. S1), indicating the pore system is not blocked due to the introduction of $\mathrm{TiO}_{2}$ into the pore channel.
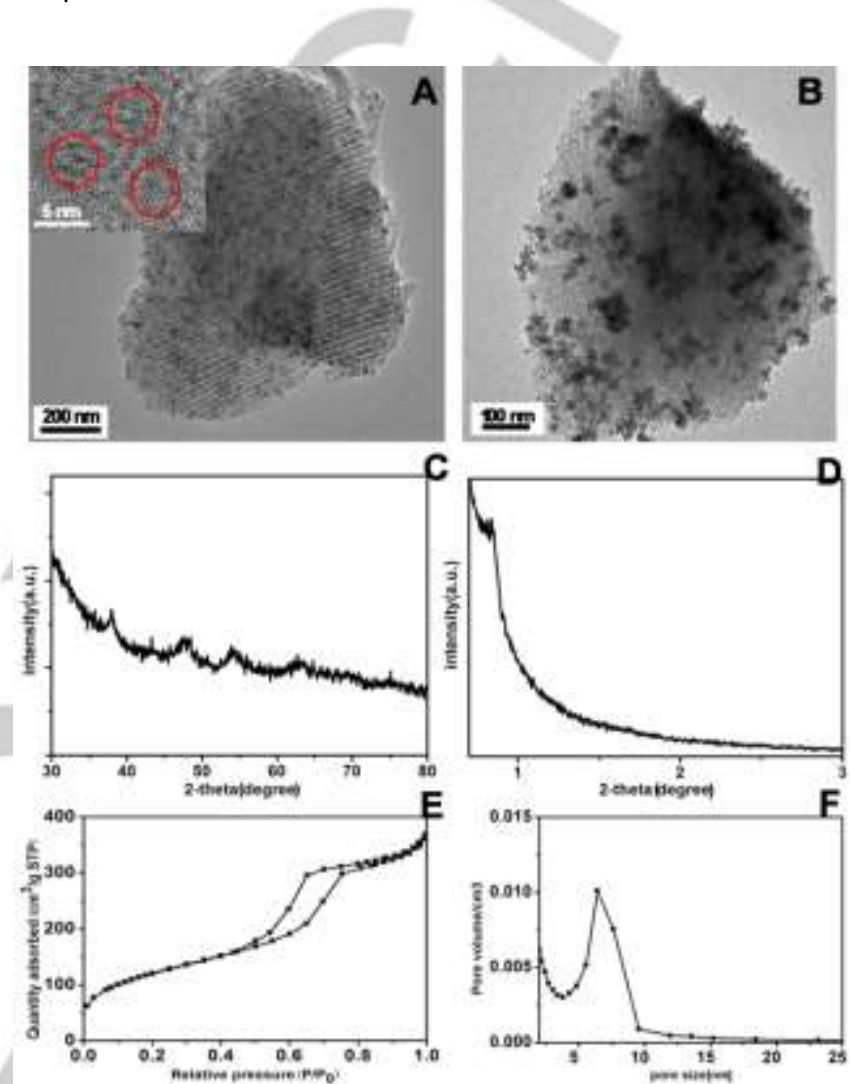

Figure 2. TEM images of Ti-CD-MS (A) and Ti-MS (B); Wide-angle XRD pattern (C), small-angle XRD pattern (D), $\mathrm{N}_{2}$ adsorption-desorption isotherms $(E)$ and pore size distribution curve (F) of Ti-CD-MS.

The element mapping by energy dispersive $\mathrm{X}$-ray (EDX) spectroscopy shows that element $\mathrm{Ti}$ is uniformly distributed on mesoporous silica accompanied with element $\mathrm{N}$, which is derived from the amide groups on CD (Fig. 3). The weight percentage contents of $\mathrm{Si}, \mathrm{Ti}$ and $\mathrm{N}$ are $37.9,19.0$ and $5.2 \mathrm{wt} \%$, respectively. Fig. 4 a shows the $\mathrm{X}$-ray photoelectron spectroscopy (XPS) for the Ti $2 p$ region of Ti-CD-MS and Ti-MS. The Ti2 $p_{3 / 2}$ and Ti2 $p_{1 / 2}$ core levels of Ti-MS appearing at 467.2 and $461.2 \mathrm{eV}$ are higher than most of the reported values ${ }^{[12]}$, which should be related to the loading environment of $\mathrm{TiO}_{2}$ nanoparticle and will be discussed together with the $\mathrm{O} 1 \mathrm{~s}$ spectrum later. The two peaks shift to 465 and $459 \mathrm{eV}$ for sample Ti-CD-MS, indicative of the increased electron density caused by the presence of $C D^{[12 a]}$. The N1s spectrum shows a broad peak centered at $401.6 \mathrm{eV}$, which is accompanied by a small peak at $396.8 \mathrm{eV}$. It is commonly accepted that the peak around $396 \mathrm{eV}$ is ascribed to the substitutional nitrogen (Ti-N) and the peak at $401.6 \mathrm{eV}$ is assigned to the oxidized Ti-N in the 
form of Ti-O-N ${ }^{[12 a, 13]}$. The C1s XPS peak can be fitted into three peaks at 284.6, 285.5 and $288.4 \mathrm{eV}$. The first peak at $284.6 \mathrm{eV}$
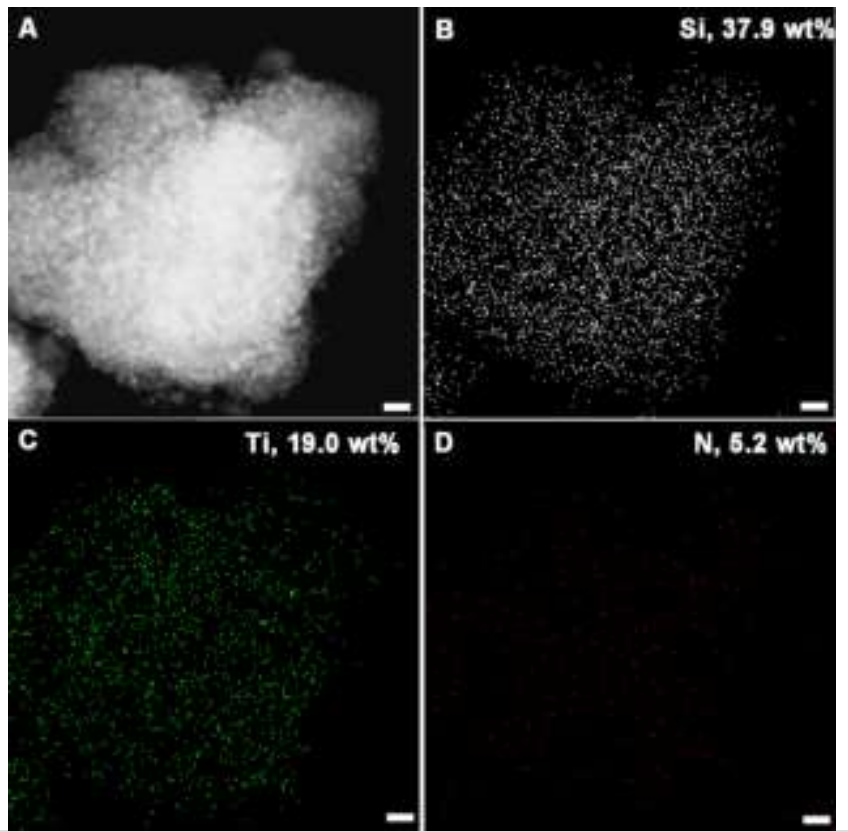

Figure 3. EDX mapping of elements $\mathrm{Si}, \mathrm{Ti}$ and $\mathrm{N}$ in Ti-CD-MS. The scale bar is $100 \mathrm{~nm}$.

is associated with the adventitious elemental carbon. The second peak at $285.5 \mathrm{eV}$ is assigned to graphitic carbon and the small peak at $288.4 \mathrm{eV}$ proves the existence of $\mathrm{C}=\mathrm{O}$ and COO ${ }^{[14]}$. Therefore, it is assumed that CD obtained from instant high-temperature pyrolysis of citric acid in the presence of amine-containing silane should be composed of a graphitic carbon core covered with an ethoxysilane shell through the linkage of amide group according to the result from UV-Vis, FTIR and C1s XPS. Moreover, the peak at $281.2 \mathrm{eV}$ corresponding to the $\mathrm{Ti}-\mathrm{C}$ bond was not observed ${ }^{[14]}$, suggesting $\mathrm{C}$ atoms in $\mathrm{CD}$ may be bonded with lattice oxygen atoms in $\mathrm{TiO}_{2}$ as $\mathrm{Ti}-\mathrm{O}-\mathrm{C}$ through substituting titanium atoms instead of replacing oxygen atoms. The fitting of $01 \mathrm{~s}$ spectrum generates three peaks at $529.6 \mathrm{eV}, 532.0 \mathrm{eV}$ and $535.2 \mathrm{eV}$. The peak at $529.6 \mathrm{eV}$ is from Ti-O-Ti and the peak at $532 \mathrm{eV}$ is ubiquitously observed from $\mathrm{TiO}_{2}$ with Ti-O-C or Ti-O-N $\mathrm{N}^{[12 a, 15]}$. The content of these two species cannot be discriminated according to the similar electronegativity of $\mathrm{C}$ and $\mathrm{N}$. The highest peak at 535.2 $\mathrm{eV}$ is related to the existence of abundant $\mathrm{O}-\mathrm{H}$ bonds in mesoporous silica, which may lead to the electron deficiency of $\mathrm{Ti}$ species loaded in the pore channel and is accordant with the $\mathrm{Ti} 2 \mathrm{p}$ XPS of Ti-MS. Based on the formation of Ti-O-N, Ti-O-C and $\mathrm{Ti}-\mathrm{N}$ bonds, it is proposed that $\mathrm{Ti}$ precursor should be chelated by $\mathrm{O}=\mathrm{CNH}_{2}$ groups in $\mathrm{CD}$, which prohibits the growth of $\mathrm{TiO}_{2}$ particle during the hydrothermal process. The finally formed $\mathrm{TiO}_{2}$ particles should be attached on CD particles through Ti-O$\mathrm{N}$ and Ti-O-C bonds and part of $\mathrm{N}$ is doped into the lattice of $\mathrm{TiO}_{2}$ in the form of $\mathrm{Ti}-\mathrm{N}$.

The UV-Vis absorption spectrum of Ti-CD-MS shows a wide absorption band from 300 to $550 \mathrm{~nm}$ (Fig. $5 \mathrm{~A}$ ). When irradiated with the visible light, Ti-CD-MS shows photocurrent much higher than CD-MS (75 times) in the absence of $\mathrm{TiO}_{2}$ and Ti-MS (30 times) in the absence of CD (Fig. 5 B). The visible-light activity can be attributed to $\mathrm{N}$ doping. However, in consideration of the
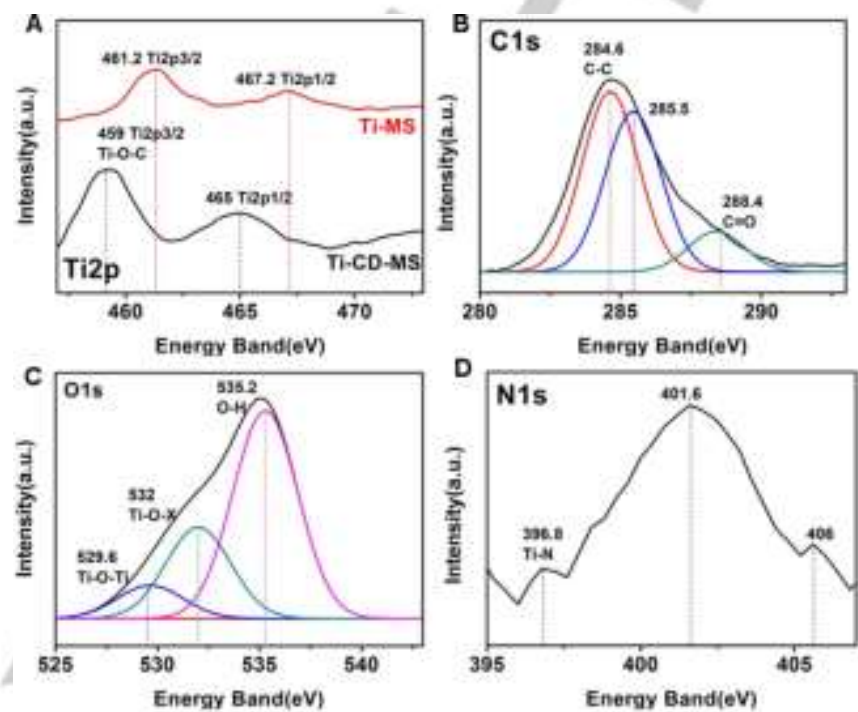

Figure 4. XPS spectra of Ti-MS and Ti-CD-MS: Ti2p (A), C1s (B), O1s (C) and N1s (D).

graphitic essence of $\mathrm{CD}$, the highest occupied molecular orbital (HOMO) and lowest unoccupied molecular orbital (LUMO) levels of $C D$ has been further calculated to reveal the possibility of photosensitization of $\mathrm{TiO}_{2}$ by neighboring $\mathrm{CD}$ bridged through $\mathrm{Ti}$ $\mathrm{O}-\mathrm{N}$ and Ti-O-C ${ }^{[16]}$. First, the energy bandgap (Eg) of CD-MS is determined to be ca. $2.51 \mathrm{eV}$ (Fig. $5 \mathrm{C}$ ) from the Tauc plot of transformed Kubelka-Munk function versus $\mathrm{h} v$ as indicated in Equation 1 ${ }^{[17]}$ :

$$
\begin{aligned}
& (\alpha h v)^{1 / 2} \text { vs. }(h v-E g) \cdots \cdots(1) \\
& H O M O=-\left[E_{o x}-E_{F c} / F c++4.5+0.24\right] e V \cdots \cdots \\
& L U M O=H O M O+E g \cdots \cdots \cdot(3)
\end{aligned}
$$

Where $\alpha$ is the absorption coefficient, $v$ is the frequency of the light, and $h$ is Planck's constant. The onset potential of oxidation $\left(E_{o x}\right)$ of CD-MS is measured as $0.11 \mathrm{~V}$ by cyclic voltammetry using saturated calomel electrode (SCE) as the reference electrode and ferrocene as the standard (Fig. $5 \mathrm{D}$ ). The potential of SCE is $0.24 \mathrm{~V}$ versus standard hydrogen electrode (SHE). The oxidation potential of ferrocene $\left(\mathrm{E}_{\mathrm{Fc} / \mathrm{Fc}+}\right)$ is $0.42 \mathrm{~V}$ versus SHE (inset, Fig. 5 D). According to the absolute potential of SHE $(-4.5 \mathrm{eV})$, the HOMO level of CD-MS is calculated to be $-4.49 \mathrm{eV}$ from the oxidation potential using Equation 2. The LUMO level of CD-MS is calculated to be $-1.98 \mathrm{eV}$ from Equation 3., allowing the electron transfer from CD-MS to anatase $\mathrm{TiO}_{2}$ with lower 
potential of conduction band $(-4.42 \mathrm{eV})$ when irradiated with visible light and resulting in the photosensitization of $\mathrm{TiO}_{2}$ by neighboring $C D^{[18]}$. Therefore, the improved photocurrent of TiCD-MS under visible light irradiation should be attributed to the co-effect of nitrogen-doping and CD-sensitization of $\mathrm{TiO}_{2}$.
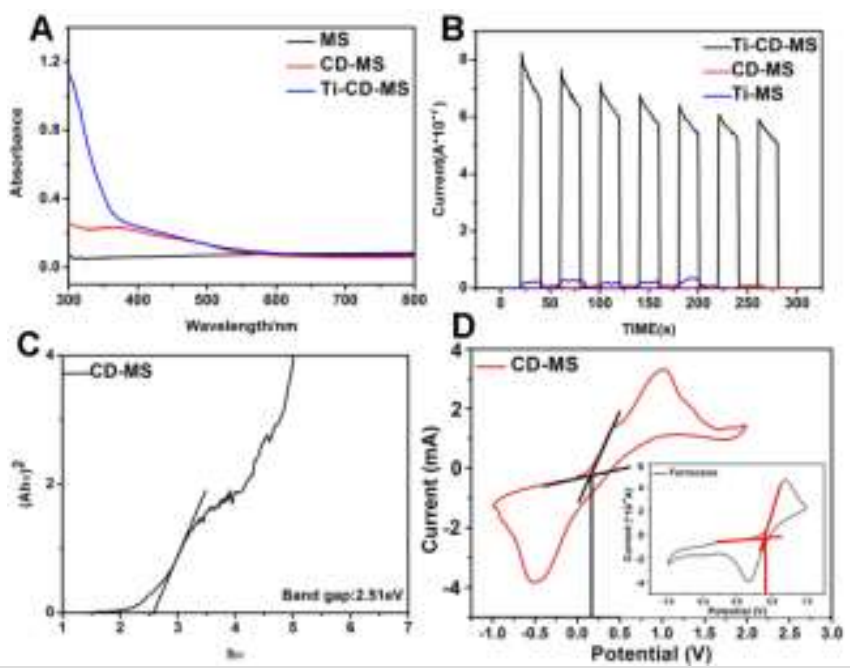

Figure 5. (A) UV-Vis spectra of Ti-CD-MS, CD-MS and MS; (B) Photocurrent of Ti-CD-MS, CD-MS and Ti-MS under visible-light irradiation; (C) Optical energy band gap and CV curve of CD-MS.
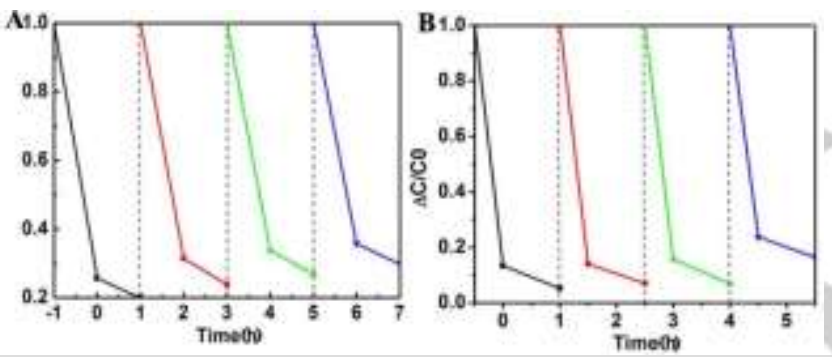

Figure 6. Adsorption-photocatalytic recycling efficiency of Ti-CD-MS for 2, 4$\operatorname{DCP}(\mathrm{A})$ and $\mathrm{AO} 7(\mathrm{~B})$.

Furthermore, it is found Ti-CD-MS shows significant adsorption capacity to 2, 4-DCP in the dark when applied as a photocatalyst, where about $75 \%$ of $2,4-\mathrm{DCP}$ can be adsorbed after the equilibrium was reached within $1.0 \mathrm{~h}$ as verified by UVVis absorption spectra (Fig. $6 \mathrm{~A}$ ). The high adsorption efficiency should be related to the incorporation of CD since Ti-MS shows less adsorption. The isoelectric point of Ti-CD-MS is at $\mathrm{pH}=4.8$ (Fig. S2), which excludes the possibility of electrostatic interaction between 2, 4-DCP and Ti-CD-MS at neutral environment. The hydrophobicity test was further carried out to identify the contribution of hydrophobic interaction due to the presence of graphitic carbon in CD. The contact angle of Ti-CDMS is about $55.23^{\circ}$ (Fig. S3), which seems incompetent to the pronounced adsorption capacity as demonstrated in Fig. 6A since the surface of Ti-CD-MS is actually hydrophilic. Currently, for the enrichment-assisted photocatalysis, hydrophobic interaction is commonly adopted. In general, hydrophobic interaction refers to the adsorption behavior of aromatic compounds without polar functional groups through weak Van der waals force. Besides hydrophobic interaction, other adsorption modes including polar- $\pi$ interactions, cation- $\pi$ and $n$ $\pi$ electron donor-acceptor (EDA) interaction have been reported $^{19]}$. 2, 4-DCP contains an electron-deficient $\pi$-system due to the presence of two electron-withdrawing $-\mathrm{Cl}$ substituents on aromatic ring. Ti-CD-MS contains amide group, where the $\mathrm{O}$ and $\mathrm{N}$ atoms should also possibly form $\mathrm{n}-\pi$ EDA interaction with the electron-deficient $\pi$-system. The binding affinity of $n-\pi$ EDA interaction is stronger than that caused by hydrophobic interaction since the bonding enthalpies involved in hydrophobic interactions are considered negligible compared with EDA interaction ${ }^{[10 c]}$. The advantage of non-hydrophobic adsorption lies in the preservation of hydrophilicity of photocatalyst, which is more feasible for the treatment of sewage water. Since $\mathrm{TiO}_{2}$ nanocrystals are anchored with $\mathrm{CD}$ through $\mathrm{Ti}-\mathrm{O}-\mathrm{N}$ and $\mathrm{Ti}-\mathrm{O}-\mathrm{C}$ bonds, the enrichment of pollutant by $C D$ can increase the local concentration of pollutant around $\mathrm{TiO}_{2}$. The absorption of 2, 4DCP keeps decreasing by further irradiated with visible light for $1 \mathrm{~h}$. The degradation of adsorbed 2, 4-DCP was verified by analyzing the elution obtained from washing photocatalyst with ethanol after the photocatalysis process with UV-Vis spectroscopy. No absorption attributed to 2, 4-DCP is detected from the elution, indicating the efficient degradation of adsorbed 2, 4-DCP. The degradation of total 2, 4-DCP in solution can be achieved by prolonging the irradiation time (4 h, Fig. S5). However, the photocatalyst can actually well recover its adsorption capacity after $1 \mathrm{~h}$ of irradiation, which still preserves its $87 \%$ of adsorption capacity at the 3rd recycle (Fig. 6A). The well preserved adsorption capacity demonstrates the good photostability of $C D$. In fact, $C D$ shows negligible variation of peak intensity even after $4 \mathrm{~h}$ of irradiation with $300 \mathrm{~W}$ of Xe lamp (Fig. S4). Moreover, to demonstrate the effect of $n-\pi$ EDA interaction on pollutant adsorption, azo dye $\mathrm{AO} 7$ also containing the electron-deficient aromatic ring was further adopted as pollutant target as shown in Fig. $6 \mathrm{~B}$. About $90 \%$ of $\mathrm{AO} 7$ is adsorbed after the equilibrium is reached within $0.5 \mathrm{~h}$. The adsorbed $\mathrm{AO} 7$ is almost completely eliminated after further irradiated with visible light for $1 \mathrm{~h}$. The recycled Ti-CD-MS can still adsorb $90 \%$ of $\mathrm{AO} 7$ and be further recycled for 3 times without obvious loss of adsorption capacity. As mentioned above, the excellent visible-light driven photocatalytic self-recycling performance should be benefitted from the co-contribution from nitrogen-doping and CD-sensitization of $\mathrm{TiO}_{2}$ (Fig. 7).

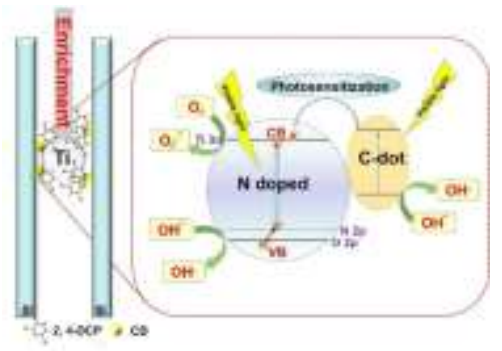


Figure 7. Schematic diagram for the visible-light driven photoatalytic selfrecycling process.

\section{Conclusions}

In conclusion, mesoporous silica embedded with amidecontaining $\mathrm{CD}$ was used for the in-situ growth of $\mathrm{TiO}_{2}$ photocalyst. Highly dispersed $\mathrm{TiO}_{2}$ nanocrystals are formed in the pore channel through the chelating between amide groups and titania precursor. Under the visible-light irradiation, Ti-CDMS shows photocurrent 75 times higher than CD-MS and 30 times higher than Ti-MS due to the co-effect of nitrogen-doping and photosensitization of $\mathrm{TiO}_{2}$ by neighboring $\mathrm{CD}$. When applied to the photocatalytic elimination of 2, 4-DCP and AO7, Ti-CDMS shows extraordinary activity through the enrichmentdegradation mode since CD can efficiently adsorb 2, 4-DCP and $\mathrm{AO} 7$ with electron-deficient aromatic ring via $n-\pi$ EDA interaction. In consideration of the high feasibility of the non-hydrophobic adsorption mode for the water decontamination, we believe the design and synthesis strategy presented here can further accelerate the development and application of enrichmentdegradation type photocatalyst for the wastewater treatment.

\section{Experimental Section}

\section{Synthesis of CD}

$C D$ was prepared through a one-step synthetic route reported previously. ${ }^{[10 a]}$ First, $10 \mathrm{ml}$ of $\mathrm{N}$-( $\beta$-aminoethyl)- $y$-aminopropyl methyldimethoxy silane (AEAPMS) was put in a $50 \mathrm{ml}$ of three-necked flask. The temperature was increased to $240{ }^{\circ} \mathrm{C}$ under nitrogen atmosphere. Then $0.5 \mathrm{~g}$ of anhydrous citric acid was quickly added into the flask. The reactants were heated at $240{ }^{\circ} \mathrm{C}$ for $1 \mathrm{~min}$ and then cooled to room temperature. The sample was obtained after washed with hexane for two times, then sealed with hexane and stored in the refrigerator.

\section{Synthesis of CD encapsulated mesoporous silica (CD-MS)}

CD-MS was prepared using $\mathrm{EO}_{20} \mathrm{PO}_{70} \mathrm{EO}_{20}(\mathrm{P} 123)$ as the template following the method described previously[5b]. P123 was first dissolved in $120 \mathrm{~mL}$ of $\mathrm{HCl}$ solution $(2.0 \mathrm{M})$ to get a homogeneous solution by vigorous stirring at $38^{\circ} \mathrm{C}$. Then, $9 \mathrm{~mL}$ of TEOS and $0.6 \mathrm{~mL}$ of CD were added and the mixture was stirred at $38^{\circ} \mathrm{C}$ for $24 \mathrm{~h}$. After that, the mixture was transferred into an autoclave and hydrothermally treated for $24 \mathrm{~h}$ at $100^{\circ} \mathrm{C}$. Then the samples were filtered, washed with deionized water, and dried at $60{ }^{\circ} \mathrm{C}$ under vacuum condition. The templates were removed by solvent-extraction method, where $50 \mathrm{mg}$ of the as-made sample, $60 \mathrm{mg}$ of sodium acetate and $5 \mathrm{ml}$ of ethanol were mixed together and stirred under refluxing at $92^{\circ} \mathrm{C}$ for $1 \mathrm{~h}$.

\section{Loading of $\mathrm{TiO}_{2}$ nanoparticles on CD-MS (Ti-CD-MS)}

$\mathrm{TiO}_{2}$ nanoparticles were loaded on CD-MS through precursorimpregnation and in-situ hydrothermal growth. First, $25 \mathrm{mg}$ of $\mathrm{Ti}\left(\mathrm{SO}_{4}\right)_{2}$ was dissolved in a solution containing $22 \mathrm{~mL}$ of ethanol and $3 \mathrm{~mL}$ of deionized water. Then $0.1 \mathrm{~g}$ of CD-MS was added under stirring. After further stirring at room temperature for $3 \mathrm{~h}$, the mixture was transferred to an autoclave and hydrothermally treated at $120^{\circ} \mathrm{C}$ for $12 \mathrm{~h}$. The obtained product was washed by deionized water and dried at $60{ }^{\circ} \mathrm{C}$ for $12 \mathrm{~h}$ under the vacuum condition. $\mathrm{TiO}_{2}$ was also in-situ loaded on $\mathrm{MS}$ through the same procedure (Ti-MS).

\section{Photocatalytic experiments}

$50 \mathrm{mg}$ of sample and $50 \mathrm{~mL}$ of 2, 4-dichlorophenol (2, 4-DCP, $10 \mathrm{mg} / \mathrm{L}$ ) or azo dye acid orange 7 (AO7, $10 \mathrm{mg} / \mathrm{L}$ ) were ultrasonically mixed. Before irradiation, the mixture was stirred for $30 \mathrm{~min}$ in the dark to reach an adsorption-desorption equilibrium. A $300 \mathrm{~W}$ high-pressure Xe lamp equipped with $420 \mathrm{~nm}$ optical filter was used as the light source. Ambient temperature was maintained during the photocatalytic reaction. At each predetermined time point, the analytical samples taken from the mixture were immediately centrifuged and then filtered through syringe filters (cellulose acetate membranes) with the size of $0.22 \mu \mathrm{m}$ to remove the photocatalysts. The filtrates were analyzed by recording variations in the UV-Vis absorption spectra of 2, 4-DCP or AO7.

\section{Characterization}

X-ray diffraction (XRD) patterns were collected on a RigakuD/MAX 2550 diffractometer using Cu Ka radiation $(40 \mathrm{kV}, 100 \mathrm{~mA}, \lambda=0.15406 \mathrm{~nm})$. $\mathrm{N}_{2}$ sorption isotherms were measured at $77 \mathrm{~K}$ by a Micromeritics ASAP 2020 analyzer. The Brunauer- Emmett-Teller (BET) method was utilized to calculate the specific surface areas (SBET) by using the Barrett-Joyner-Halenda (BJH) model, the pore volumes and pore size distributions were derived from the adsorption branches of isotherms. The transmission electron microscopy (TEM) was conducted on a JEM 2000EX microscope. Fourier Transform Infrared (FT-IR) spectra were recorded from a Nicolet FTIR spectrometer (Nicolet Magna 550). UV-Vis absorption or diffuse reflection spectra were performed on a Scan UV-Vis spectrophotometer (SHIMADZU, UV-2450), with $\mathrm{BaSO}_{4}$ as the reflectance sample. Zata-potential vs. $\mathrm{pH}$ curves were analyzed by a Malvern Zetasizer Nano-ZS instrument (ZEN3600, Malvern Instruments), using $0.1 \mathrm{M}$ of $\mathrm{NaOH}$ and $\mathrm{HCl}$ to adjust the $\mathrm{pH}$ value, and $0.1 \mathrm{M}$ of $\mathrm{NaCl}$ to maintain a high background-electrolyte concentration. For the electrochemical analysis, the counter, reference and working electrodes are Pt electrode, saturated calomel electrode (SCE) and FTO glass coated with thin film of tested sample, respectively. The supporting electrolyte is a mixed solution of $(2.5 \mathrm{mM}) \mathrm{K}_{3} \mathrm{Fe}(\mathrm{CN})_{6}$ and $(0.1 \mathrm{M}) \mathrm{KCl}$, where $\mathrm{K}_{3} \mathrm{Fe}(\mathrm{CN})_{6}$ is used as an internal standard. The electrode potential is $0.24 \mathrm{~V}$ versus normal hydrogen electrode (NHE). The scan rate is $100 \mathrm{mV} / \mathrm{s}$.

\section{Acknowledgements}

This work has been supported by the National Nature Science Foundation of China (U1407102, 21322508, 21210004, 21173077 and 21377038), the National Basic Research Program of China (973 Program, 2013CB632403, 2013CB934100, 2012CB224805), the Science and Technology Commission of Shanghai Municipality (14ZR1410700 and 14230710500), the Research Fund for the Doctoral Program of Higher Education (20120074130001), the Program for New Century Excellent Talents in University (NCET) and the Fundamental Research Funds for the Central Universities. 
Keywords: Carbon dot $•$ Mesoporous silica $\cdot \mathrm{TiO}_{2} \cdot$ Adsorption • Degradation

[1] a) A. Fujishima, K. Honda, Nature 1972, 37-38; b) S. N. Frank, A. J. Bard, J. Am. Chem. Soc. 1977, 99, 303-304; c) S. N. Frank, A. J. Bard, J. Phys. Chem. 1977, 81, 1484-1488; d) W. Wei, C. Yu, Q. Zhao, G. Li, Y. Wan, Chem. Eur. J. 2013, 19, 566-577; e) P. A. DeSario, J. J. Pietron, D. H. Taffa, R. Compton, S. Schünemann, R. Marschall, T. Brintlinger, R. Stroud, M. Wark, J. C. Owrutsky, J. Phys. Chem. C 2015 DOI: 10.1021/acs.jpcc.5b04013

[2] a) M. R. Hoffmann, S. T. Martin, W. Choi, D. W. Bahnemann, Chem. Rev. 1995, 95, 69-96; b) A. Wold, Chem. Mater. 1993, 5, 280-283.

[3] a) W. Q. Fang, J. Z. Zhou, J. Liu, Z. G. Chen, C. Yang, C. H. Sun, G. R. Qian, J. Zou, S. Z. Qiao, H. G. Yang, Chem. Eur. J. 2011, 17, 1423 1427; b) G. Hodes, Adv. Mater. 2007, 19, 639-655.

[4] a) R. Asahi, T. Morikawa, T. Ohwaki, K. Aoki, Y. Taga, Science 2001 293, 269-271; b) D. Y. Qi, L. Lu, Z. Xi, L. Wang, J. Zhang, Appl. Catal. B Environ. 2014, 160: 621-628.

[5] a) C. Kresge, M. Leonowicz, W. Roth, J. Vartuli, J. Beck, Nature 1992 , 359, 710-712; b) D. Zhao, J. Feng, Q. Huo, N. Melosh, G. H Fredrickson, B. F. Chmelka, G. D. Stucky, Science 1998, 279, 548-552; c) Y. Li, B. P. Bastakoti, M. Imura, S. M. Hwang, Z. Sun, J. H. Kim, S. X. Dou, Y. Yamauchi, Chem. Eur. J. 2014, 20, 6027-6032; d) X. Tan, L. Lu, L. Wang, J. Zhang, Eur. J. Inorg. Chem. 2015, DOI: 10.1002/ejic.201500267; e) X. Yan, L. Wang, D. Qi, J. Lei, B. Shen, T. Sen, J. Zhang, RSC Adv. 2014, 4, 57743-57748; f) L. Jiang, L. Wang, J. Zhang, Chem. Commun. 2010, 46, 8067-8069.

[6] a) L. Z. Wang, L. Jiang, C. C. Xu, J. L. Zhang, J. Phys. Chem. C 2012, 116, 16454-16460; b) L. Lu, F. Teng, D. Qi, L. Wang, J. Zhang, Appl. Catal. B: Environ. 2015, 163, 9-15. c) Z. Jiao, Y. Zhang, T. Chen, Q. Dong, G. Lu, Y. Bi, Chem. Eur. J. 2014, 20, 2654-2662; d) S. Rodrigues, K. T. Ranjit, S. Uma, I. N. Martyanov, K. J. Klabunde, Adv. Mater. 2005, 17, 2467-2471.

[7] a) D. Yang, J. Zhao, H. Liu, Z. Zheng, M. O. Adebajo, H. Wang, X. Liu, H. Zhang, J. C. Zhao, J. Bell, Chem. Eur. J. 2013, 19, 5113-5119; b) X Sun, H. Liu, J. Dong, J. Wei, Y. Zhang, Catal. Lett. 2010, 135, 219-225.
[8] a) A. Walcarius, L. Mercier, J. Mater. Chem. 2010, 20, 4478-4511; b) X Yang, Q. Guan, W. Li, J. Environ. Manage. 2011, 92, 2939-2943.

[9] X. Qian, T. Kamegawa, K. Mori, H. Li, H. Yamashita, J. Phys. Chem. C 2013, 117, 19544-19551.

[10] a) F. Wang, Z. Xie, H. Zhang, C.-Y. Liu, Y.-G. Zhang, Adv. Funct. Mater. 2011, 21, 1027-1031; b) Z. Xie , F. Wang, C.-Y Liu, Adv. Mater 2012, 24, 1716-1721; c) J. Lei, L. Yang, D. Lu, X. Yan, C. Cheng, Y. Liu, L. Wang, J. Zhang, Adv. Opt. Mater. 2015, 3, 57-63; d) L. Wang, C Cheng, S. Tapas, J. Lei, M. Matsuoka, J. Zhang, F. Zhang, J. Mater. Chem. A 2015, 3, 13357-13364.

[11] a) R. J. White, R. Luque, V. L. Budarin, J. H. Clark, D. J. Macquarrie, Chem. Soc. Rev. 2009, 38, 481-494; b) Z. K. Sun, B. Sun, M. H. Qiao J. Wei, Q. Yue, C. Wang, Y. H. Deng, S. Kaliaguine, D. Y. Zhao, J. Am. Chem. Soc. 2012, 134, 17653-17660; c) S. Wang, Q. F. Zhao, H. M. Wei, J. Q. Wang, M. Cho, H. S. Cho, O. Terasaki, Y. Wan, J. Am. Chem. Soc. 2013, 135, 11849-11860.

[12] a) Y. Cong, J. L. Zhang, F. Chen, M. Anpo, J. Phys. Chem. C 2007 111, 6976-6982; b) M. Xing, J. Zhang, F. Chen, Appl. Catal. B: Environ. 2009, 89, 563-569

[13] R. Asahi, T. Morikawa, T. Ohwaki, K. Aoki, Y. Taga, Science, 2001, 293, 269-271.

[14] a) J. Zhang, Y. Wu, M. Xing, S. A. K. Leghari, S. Sajjad, Energ. Enviro. Sci. 2010, 3, 715-726; b) Y. Wu, M. Y. Xing, J. Zhang, J. Hazard. Mater. 2011, 192, 368-373.

[15] a) Y. Nosaka, M. Matsushita, J. Nishino, A. Y. Nosaka, Sci. Technol. Adv. Mater. 2005, 6, 143-148; b) G. Yang, Z. Jiang, H. Shi, T. Xiao, Z. Yan, J. Mater. Chem. 2010, 20, 5301-5309; c) A. Le Goff, V. Artero, B. Jousselme, P. D. Tran, N. Guillet, R. Métayé, A. Fihri, S. Palacin, M. Fontecave, Science 2009, 326, 1384-1387.

[16] a) C. Ma, L. Zhang, J. Zhou, X. Wang, B. Zhang, Y. Cao, P. Bugnon, M. Schaer, F. Nüesch, D. Zhang, J. Mater. Chem. 2002, 12, 3481-3486; b) Y. J. Chang, T. J. Chow, J. Mater. Chem. 2011, 21, 3091-3099.

[17] L. Yoong, F. K. Chong, B. K. Dutta, Energy 2009, 34, 1652-1661.

[18] J. S. Lee, K. H. You, C. B. Park, Adv. Mater. 2012, 24, 1084-1088

[19] M. Keiluweit, M. Kleber, Environ. Sci. Technol. 2009, 43, 3421-3429. 


\section{Entry for the Table of Contents}

Layout 1:

\section{FULL PAPER}

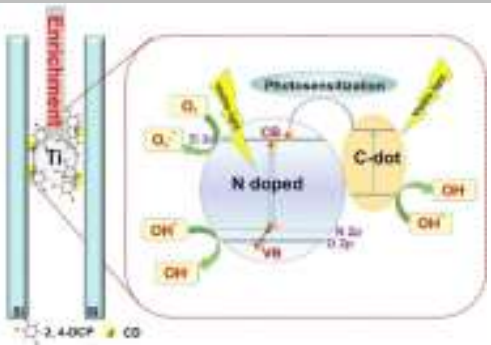

Lingzhi Wang, ${ }^{1,2}$ Chen Cheng ${ }^{1}$, Tapas Sen, ${ }^{3}$ Juying Lei, ${ }^{1}$ Jinlong Zhang, ${ }^{1 *}$ Fan Zhang ${ }^{2 *}$ and Dongyuan Zhao ${ }^{2}$

Page No. - Page No.

Carbon-dot Sensitized and $\mathrm{N}$-doped $\mathrm{TiO}_{2}$ in Mesoporous Silica for Water Decontamination through Non-hydrophobic Enrichment-Degradation Mode

$1 \quad$ Key Lab for Advanced Materials and Institute of Fine Chemicals, East China University of Science and Technology, Shanghai, 200237, P. R. China

E-mail: wlz@ecust.eud.cn; jlzhang@ecust.edu.cn

2 Department of Chemistry and Laborotary of Advanced Materials, iChEm (Collaborative Innovation Center of Chemistry for Energy Materials), State Key Laboratory of Molecular Engineering of Polymers, Fudan University, Shanghai 200433, P. R. China E-mail: zhang_fan@fudan.edu.cn

3 Center for Materials Science, Institute of Nanotechnology and Bioengineering, School of Forensic and Investigative Sciences, University of Central Lancashire, Preston

Supporting information for this article is given via a link at the end of the document. 